\title{
Face Recognition Based on Viola-Jones Face Detection Method and Principle Component Analysis (PCA)
}

\author{
Suhad Ibrahim Mohammed, Noor Abdulmuttaleb Jaafar, Kilan Mohammed Hussien \\ Suhad698@gmail.com, hassantalal81@gmail.com,kailan_moh@yahoo.com
}

\begin{abstract}
Face recognition is one of the most important research fields of the last two decades. This is due to the actual use of this technology in automatic detection and monitoring systems. Face attribute and features recognition from images is still a challenge. In this paper, face image recognition is proposed upon local face image rather than focusing on the whole image recognition by applying preprocessing techniques and Viola-Jones method. Principal Component Analysis (PCA) method is used in order to extract the needed features. Experiments show satisfied and more accurate results achieved by the proposed system comparing to the existing systems.
\end{abstract}

Keywords_-Face image Recognition; PCA; Viola-Jones; MUCT; Feature Extraction.

\section{INTRODUCTION}

Face recognition is turning into a promising instrument for electronic mixed media content assessment [1]. The human face plays an important role in our community interaction, turning over people's identity. Using the human face as a solution to security, biometric face recognition tools have received significant attention in the past several years due to their potential for a large variety of applications in both rule enforcement and non-rule enforcement [2].

Together with the most recent major fear monger issue in the common world, there has been venturing by step significant interests in the improvement of smart observing cameras that can consequently find and perceive referred to crooks and also flawed characters. Due to such uncertain circumstances, people are starting to look for help from PCs frameworks to help during the time spent distinguishing proof in an area of appearances in everyday scenes [3].

This procedure incorporates the extraction of highlights of the question picture and choosing the most surmised (coordinated) picture from the database of the images of countenances of people. The highlights of each picture in the database are as of now separated and these highlights are utilized for perceiving an obscure face.

\section{LITERATURE SURVEY}

Principle component analysis (PCA) has been contemplated in the previous years and wound up as one of the real fields in confront acknowledgment. This outcome entrenched ways to deal with utilizing eigenvector and eigenvalue in acknowledgment frameworks. This strategy, hypothetically, prompts an expanded capacity to recognize faces in troublesome conditions.

Face recognition is a procedure in which a man is confirmed or distinguished from a computerized image or a video outline. It is a prominent technique for individual recognizable proof because of its non-obtrusive trademark and giving worthy acknowledgment precision. Face recognition is a fascinating and testing issue and it affects imperative applications in numerous territories, for example, distinguishing proof for law authorization, validation for saving money and security framework 
access, and individual recognizable proof among others. Face acknowledgment, then again, still faces numerous issues on its acknowledgment exactness because of an ecological condition, particularly encompassing illumination [4].

A few techniques for face recognition and detecting have been accounted by various analysts. Some of these works are demonstrated as follows:

Yuan L. [2013] proposed a crossover strategy for PCA. Firstly, the technique for eight eyes division was acquainted with extricating the compelling zone of outward appearance picture; at that point, PCA removed the worldwide grayscale highlight of the entire outward appearance picture and decreased the information estimate in the meantime. LBP removed the nearby neighbor surface element of the mouth area [5].

Singh [2013] suggested a face recognition system that distinguishes the face area from a photo of one or numerous people together. The Viola-Jones object detection is the first framework to provide competitive object detection and it can be trained to detect a mixture of object classes. The proposed display utilizes the connection shown by the goal of the face recognition. The face acknowledgment process can identify the individual among the database of countenances without knowing some other insights about the individual particular. The proposed confront location and acknowledgment model can be sent anyplace it is required [6].

Barnouti, NawafHazim [2016] executed a programmed confront acknowledgment framework in light of appearance-based strategies. Face image recognition applying algorithm of face detection is ViolaJones technique to identify right faces of every person in the database. Face detection of Viola-Jones algorithm indicates raising identification accuracy rates PCA-LDA execution, which was effective. Square Euclidean Distance is utilized to gauge the separation between two pictures, which prompts discovering images likeness. GrimaceandFace 94 databases applying diverse of testing and preparing pictures indicate high acknowledgment accuracy rates, while MUCT database demonstrates low acknowledgment rates. The acknowledgment time was satisfactory and takes a few moments. The outcomes demonstrate expanding in acknowledgment rates when increasing the quantity of preparing images [7].

Deshpande, Narayan [2017] used an effective approach to confront acknowledgment and detection utilizing Viola-Jones, a combination of PCA and ANN methods. The execution of the proposed strategy is contrasted with other existing face acknowledgment strategies and it was shown that better precision in acknowledgment is achieved with the proposed technique. Face Detection and Recognition utilizing Viola-Jones calculation of ANN and PCA discovery and recognition assumes an indispensable part in an extensive variety of utilizations. In a large portion of the applications, a high rate of precision in distinguishing a man is wanted thus the proposed strategy can be considered in correlation with the current methods [8].

Tikoo, Smriti [2017]. At last we can finish up by expressing that there is simply one more technique which is utilized for facial acknowledgment, wherein a facial picture was caught and the fundamental strides to prepare and process the picture were completed. The preparation was performed 5-10 times to get fitting outcomes. This system has been utilized for preparing different examples and it turns out to be sufficiently precise in giving outcomes. The future degree should be possible to mechanize the whole procedure or part of it [9].

\section{VIOLA - JONES DETECTION}

The system of Viola-Jones question detection [10] is viewed as the main structure of protest identification that gives focused rates to recognize the protest progressively. An assortment of question classes has been prepared to recognize an assortment of protest utilizing Haar-like element. It was 
inspired principally by the issue of face location. Fig. 1 demonstrates some question identification utilizing Viola and Jones.
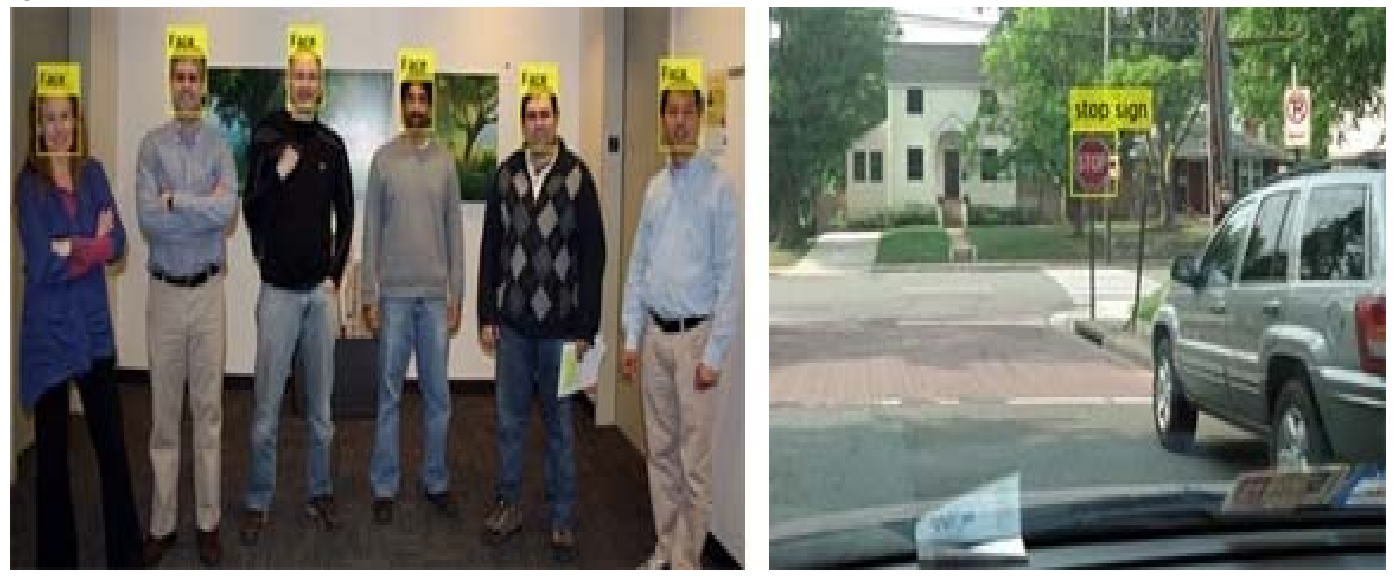

Fig.1.VIOLA - JONES OBJECT DETECTION [11]

\section{FEATURE EXTRACTION}

To classify a face in the image, features must first be extracted out of the face image. These features of human faces are either local or global. Feature extraction extracts the best discriminate features of the face image that are not susceptible to unpredictable environmental differences such as contrasts in scale, illumination, pose and facial image expressions 12][13]. Here, the PCA used feature extraction of the face image recognition for the goal of feature extraction. The PCA method is an approach adopted for face image recognition. The covariance matrix is utilized to extract the data about the feature vector value. Eigen-faces statistically are the principal components to split the face into feature value. The differences among some faces are computed by applying these vectors' values. The linear combination of the highest Eigenvalues has represented the faces. So, the face image recognition can be seen as a linear combination of the Eigen-faces [14][15].

Keeping a large amount will be computationally challenging, while taking the number of Eigen-faces to maintain is a very significant matter because keeping a small set will not detect all the variances in the dataset [16].

\section{THE PROPOSED SYSTEM}

The proposed system of face image recognition apply a person face image recognition taken from database MUCT of each person from an input and recognizes the desired person from his/her face image. The proposed system has four main steps which are: firstly, applying pre-processing by converting color image into grayscale and secondly, by viola-jones for person face detection, which divids the face into four segments of each person including eyes, nose, mouth and face segments, and thirdly, using PCA for features extraction, and finally face recognition. Furthermore, Millsboro/University of Cape Town (MUCT) database is used in this work to evaluate the system performance. Fig. 2 depicts the process of the proposed system.

\section{A. FACE IMAGE DATABASE}

Many face databases are possible for the face recognition study. Subjects were not asked to display any particular facial expression; in practice, this means that most of them were photographed with a neutral expression or a smile. All subjects were 18 or more years of age. MUCT is an image of $480 * 640$ database, which is expressed by different lighting, age, and ethnicity database including 240 faces. Each person is an image applying five webcams, which perform the database applied to an 
effect, which demands many happening features of the person. Fig. 3 depicts person images of MUCT database.

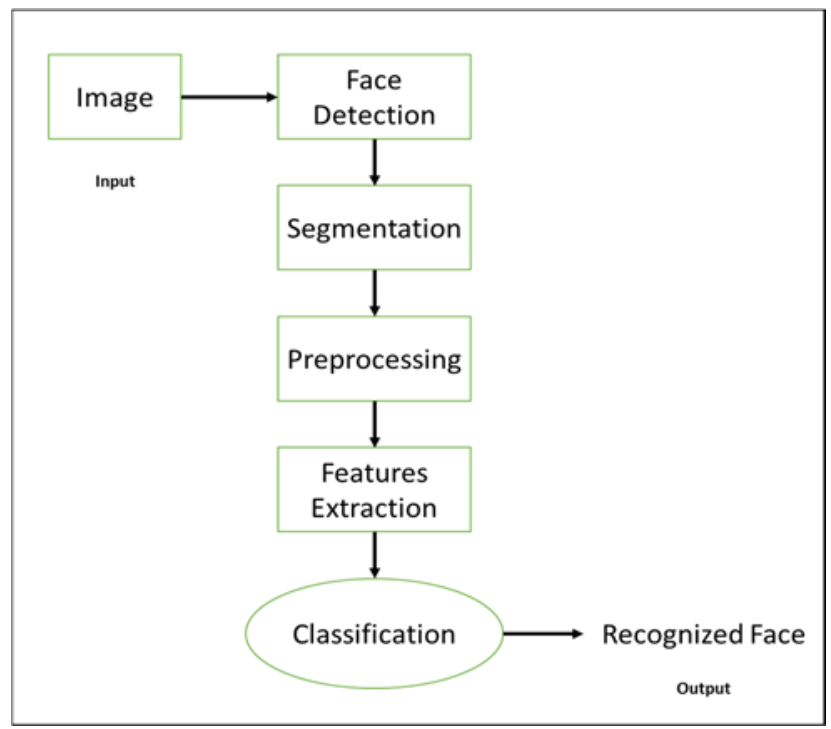

FIG. 2. A DIAGRAM OF THE PROPOSED SYSTEM

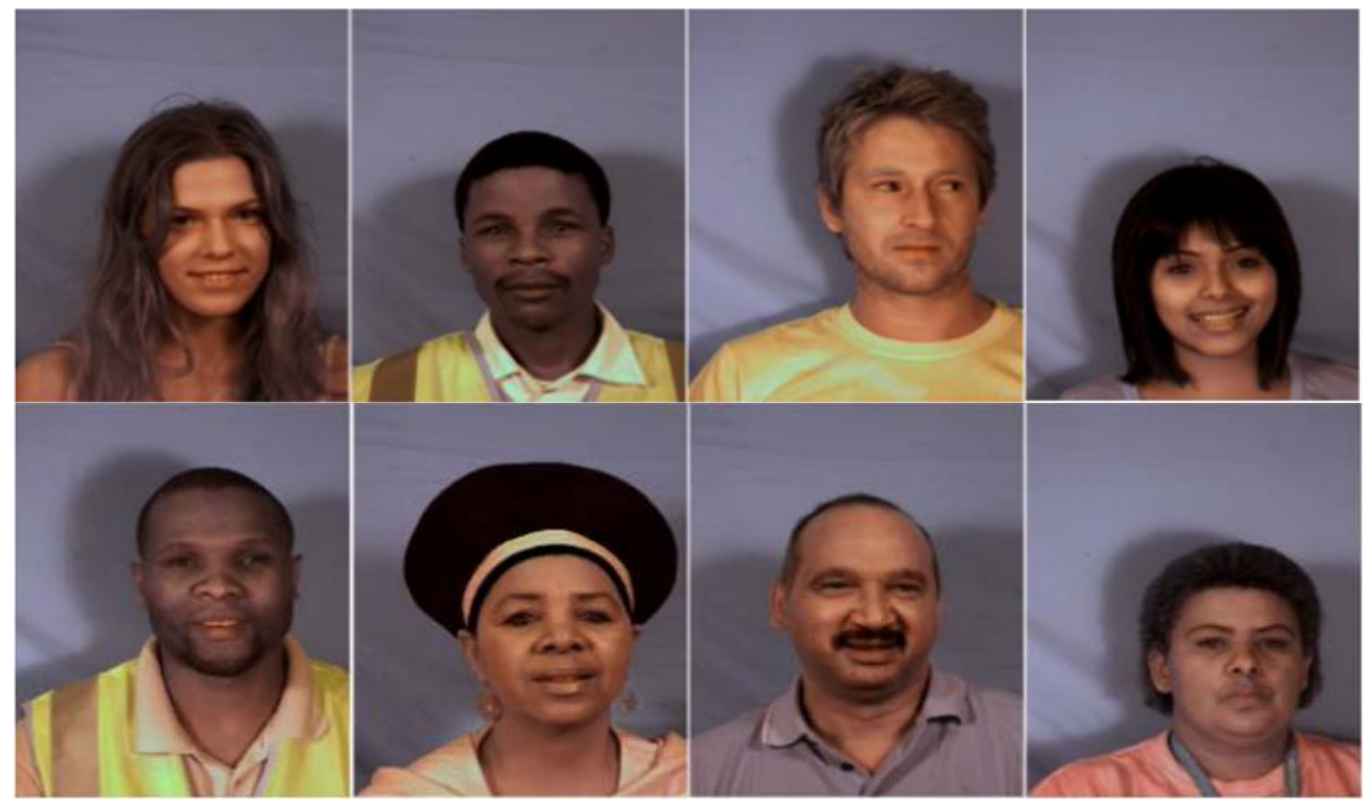

Fig.3. SAMPLES OF PERSON IMAGES FROM THE MUCT DATABASE

\section{B. FACE IMAGE DETECTION}

Face image detection is the first step in the detection system. The face detection method, which uses the Viola-Jones, is split into four main parts (Integral image, haar-like feature, classifier learning with AdaBoost and attention cascade structure) that are used to establish a strong face image detection that can be applied in the face image recognition application. Figure 4 displays the algorithm of face detection. 


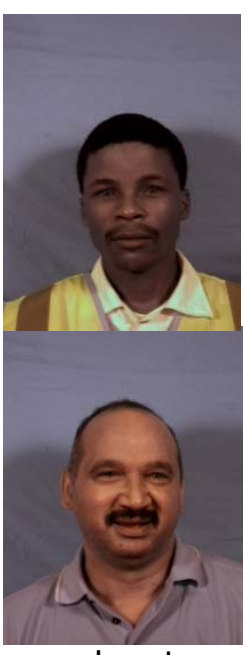

Input

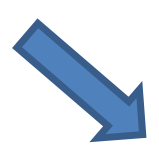

\section{Cascade Algorithm}

Fig. 4. CASCADE ALGORITHM OF VIOLA-JONES METHOD

\section{SEGMENTATION}

In the proposed system, the segmented images are passed to the next stage of the system. Haar cascade method is used for segmenting the face image into three parts; the eyes, nose, and the mouth is segmented for each person face. Figure 5 shows the face segmentation of each person.

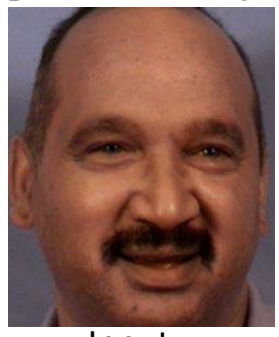

Input
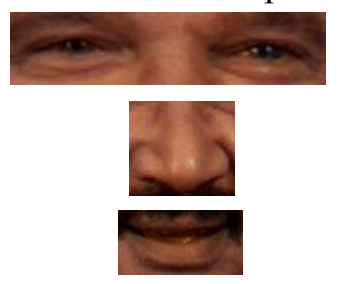

Output

FIg.5. FACE SEGMENTATION

\section{FEATURE EXTRACTION}

PCA is used to decrease the dimensional face image, mainly Eigen-face is the Eigenvector selected from PCA Eigen-faces, as a small group of distinctive images that are applied to describe the variation between face images. In gender classification, each training phase of face image recognition is converted into a face vector by multiple variance images to get the covariance matrix. Eigenfaces (Eigenvectors associated with Eigenvalues) are constructed, which represent various features of face image recognition, as shown in the algorithm below. 


\section{Algorithm PCA - Faces Feature Extraction}

Step_1 : Input: Transform face Images

Step_2 : Begin

Step_3 : Store transform face images in matrix $(\mathrm{X})$

Step_4: Calculate mean vector of $(1 * \mathrm{M})$, each element of mean is the average of the corresponding column of $x$ matrix.

Sub_X is a matrix $\left(M^{*} N\right)$, each row represents the centered zero mean of faces vectors, and they are calculated as:

For each $i^{\text {th }}$ row of X Sub_ $X_{i j}=X_{i j}-$ Mean_ $X_{i j}$

For $\mathrm{j}=1,2, \ldots \mathrm{N}$.

Step_5: Compute variance to get a modified ensemble of vectors $\mathrm{X}=\{\mathrm{Xn}, \mathrm{n}=1, . . \mathrm{N}\}$ with $\mathrm{Xn}=\mathrm{Xn}-\mathrm{E}(\mathrm{X})$

Step_6: Compute covariance matrix $\mathrm{X} \_\mathrm{Cov}=\mathrm{Sub} \_\mathrm{X} * \mathrm{Sub} \mathrm{X}^{\mathrm{T}}$ and size of $\mathrm{X} \_\mathrm{Cov}$ is $\left(\mathrm{M}^{*} \mathrm{M}\right)$.

Step_7: Extract Eigen faces, select eigenvector to extract Eigen_faces when: Diagonal of eigenvalue matrix $>=1$

Eigen_faces $=$ variance $*$ selected eigenvector

\section{Step_8 : Output: Eigen faces}

End.

\section{Experimental data and results}

The proposed system is implemented by MATLAB software and under Microsoft Windows environment. The (MUCT) database was applied to evaluate the accuracy rate of the system performance. The dataset uses 60 images of four segments for the training and testing phases. The Viola-Jones method achieved high detection accuracy rate for face detection of each person in the dataset. All images are cropped and detected in the databases.

To measure the distance, the Square Euclidean Distance between images is used for classifying the right face.

In this proposed system, the result used three images of 20 persons and applied six segments types, namely; eyes, mouth, nose, eyes with a nose, eyes with nose and face segments, in training images 60 of each segment, and testing phase segments 60 from dataset of 240 images of 20 persons, for each person 12 images.

In the proposed system, the result of performance accuracy of segment eyes is $100 \%$, segment mouth is $11.11 \%$, segments nose is $70.37 \%$, eyes with the nose is $38.88 \%$, eyes with a mouth is $84.58 \%$ and the face is $94.79 \%$, representing the accuracy rate performance of each segment. 
TABLE.1. PERFORMANCE OF SIX SEGMENTS of THREE IMAGES of 20 PERSONS

\begin{tabular}{|lllll|}
$\begin{array}{l}\text { Experiment } \\
\text { Set }\end{array}$ & Segment type & $\begin{array}{l}\text { Number of } \\
\text { train images }\end{array}$ & $\begin{array}{l}\text { Number of } \\
\text { test images }\end{array}$ & Performance(\%) \\
\hline $\mathbf{1}$ & Eye & 60 & 60 & 100 \\
\hline $\mathbf{2}$ & Mouth & 60 & 60 & 11.11 \\
\hline $\mathbf{3}$ & Nose & 60 & 60 & 70.37 \\
\hline $\mathbf{4}$ & Eye and nose & 60 & 60 & 38.88 \\
\hline $\mathbf{5}$ & Eye and mouth & 60 & 60 & 84.58 \\
\hline $\mathbf{6}$ & Face & 60 & 60 & 94.79 \\
\hline
\end{tabular}

In this proposed system, the result used 12 images of 20 persons and applied six segments types, namely; eyes, mouth, nose, eyes with nose, eyes with nose and face segments, in training images 240 of each segment, and testing phase segments 240 from the dataset of 240 images of 20 person, for each person 12 images .

In the proposed system, the result of performance accuracy of segment eyes is $95.33 \%$, segment mouth is $52.91 \%$, segments nose is $60.54 \%$, eyes with nose is $24.51 \%$, eyes with mouth is $65.72 \%$ and face is $81.48 \%$, repressing the accuracy rate performance of each segment.

TABLE.2. PERFORMANCE OF SIX SEGMENTS of 12 IMAGES of 20 PERSONS

\begin{tabular}{|c|c|c|c|c|}
\hline $\begin{array}{l}\text { Experiment } \\
\text { Set }\end{array}$ & Segment type & $\begin{array}{l}\text { Number of } \\
\text { train images }\end{array}$ & $\begin{array}{l}\text { Number of } \\
\text { test images }\end{array}$ & Performance(\%) \\
\hline 1 & Eye & 240 & 240 & 95.33 \\
\hline 2 & Mouth & 240 & 240 & 52.91 \\
\hline 3 & Nose & 240 & 240 & 60.54 \\
\hline 4 & Eye and nose & 240 & 240 & 24.51 \\
\hline 5 & Eye and mouth & 240 & 240 & 65.72 \\
\hline 6 & Face & 240 & 240 & 81.48 \\
\hline
\end{tabular}

\section{CONCLUSION}

Numerous techniques are adopted in order to perform the valid recognition results. To race up the performance of the system, Viola-Jones algorithm was applied. The Haar cascade approach has been used for segmentation and PCA was proposed as feature extraction using distance square for classification. Within adopted schemes, the proposed system performed supplied results comparing to the current systems. The system applied different segments and got different results of accuracy rate performance.

\section{REFERENCES}

[1] Turk, Matthew A., and Alex P. Pentland. "Face recognition using eigenfaces." Computer Vision and Pattern Recognition, 1991. Proceedings CVPR'91., IEEE Computer Society Conference on. IEEE, 1991

[2] Chen, Li-Fen, et al. "A new LDA-based face recognition system which can solve the small sample size problem." Pattern recognition 33.10 (2000): 1713-1726

[3]Wiskott, Laurenz, et al. "Face recognition by elastic bunch graph matching." IEEE Transactions on pattern analysis and machine intelligence 19.7 (1997): 775-779. 
[4] Wagner, Andrew, et al. "Toward a practical face recognition system: Robust alignment and illumination by sparse representation." IEEE Transactions on Pattern Analysis and Machine Intelligence 34.2 (2012): 372-386

[5] Yuan, L. U. O., Cai-ming Wu, and Yi Zhang. "Facial expression feature extraction using hybrid PCA and LBP." The Journal of China Universities of Posts and Telecommunications 20.2 (2013): 120-124.

[6] Singh, Ranjeet, and Mandeep Kaur. "Face Recognition and Detection using Viola-Jones and Cross Correlation Method . 2013.

[7] Barnouti, NawafHazim, et al. "Face Detection and Recognition Using Viola-Jones with PCA-LDA and Square Euclidean Distance." International Journal of Advanced Computer Science and Applications (IJACSA) 7.5 (2016).

[8]Deshpande, Narayan T., and S. Ravishankar. "Face Detection and Recognition using Viola-Jones algorithm and Fusion of PCA and ANN." Advances in Computational Sciences and Technology 10.5 (2017): 1173-1189

[9] Tikoo, Smriti, and Nitin Malik. "Detection of Face using Viola Jones and Recognition using Back Propagation Neural Network." arXiv preprint arXiv:1701.08257 (2017).

[10] Paul Viola, Michael J.Jones, Robust Real-Time Face Detection , 2002.

[11] Viola, Paul, and Michael Jones. "Rapid object detection using a boosted cascade of simple features." Computer Vision and Pattern Recognition, 2001.CVPR 2001.Proceedings of the 2001 IEEE Computer Society Conference on.Vol. 1. IEEE, 2001.

[12] Hussein Rady, "Face Recognition using Principle Component Analysis with Different Distance Classification" (El-Shorouk Academy, Higher Institute for computer \& Information Technology, Egypt), IJCSNS International Journal of Computer Science and Network Security, VOL.11 No.10, October 2011.

[13] Yang, Jian, et al. "Two-dimensional PCA: a new approach to appearance-based face representation and recognition." IEEE transactions on pattern analysis and machine intelligence 26.1 (2004): 131-137.

[14] Cheriyadat, Anil, and L. Mann Bruce. "Why principal component analysis is not an appropriate feature extraction method for hyperspectral data." Geoscience and Remote Sensing Symposium, 2003.IGARSS'03.Proceedings.2003 IEEE International.Vol. 6. IEEE, 2003.

[15] Yang, Jian, et al. "Two-dimensional PCA: a new approach to appearance-based face representation and recognition." IEEE transactions on pattern analysis and machine intelligence 26.1 (2004): 131-137.

[16] Kim, Kwang In, Keechul Jung, and Hang Joon Kim. "Face recognition using kernel principal component analysis." IEEE signal processing letters 9.2 (2002): 40-42. 